\section{ORIGINAL RESEARCH}

\author{
C.T. Whitlow \\ B.J. Mussat-Whitlow \\ C.W.T. Mattern \\ M.D. Baker \\ P.P. Morris
}

\title{
Sacroplasty versus Vertebroplasty: Comparable Clinical Outcomes for the Treatment of Fracture- Related Pain
}

BACKGROUND AND PURPOSE: Little is known about the long-term clinical outcomes of sacroplasty, a relatively new minimally invasive percutaneous procedure for the treatment of sacral insufficiency fractures. The first purpose of the present study, therefore, was to investigate the effects of sacroplasty on pain, mobility, and activities of daily living (ADLs). A second purpose was to compare clinical outcomes of sacroplasty with those of vertebroplasty, a similar but more established procedure.

MATERIALS AND METHODS: A retrospective case series of 12 patients who had a sacroplasty and a control group of 21 patients who had undergone a vertebroplasty was conducted. A 12-item questionnaire and subsequent telephone interview requested each patient to rate the intensity of pain, as well as the ability to ambulate and perform ADLs, before sacroplasty or vertebroplasty, and at the time of the interview.

RESULTS: There was a statistically significant decrease in overall self-reported pain, as well as an increase in self-reported ability to ambulate and perform ADLs after sacroplasty or vertebroplasty. These improvements were equivalent, regardless of which procedure the patient received.

CONCLUSION: The present study suggests that the treatment of sacral insufficiency fractures with sacroplasty produces relatively long-lasting improvements in pain, mobility, and the ability to perform ADLs. These data also suggest that the clinical outcomes of sacroplasty are comparable with those of vertebroplasty, an accepted and more routinely performed procedure.

$\mathbf{S}^{a}$ acral insufficiency fractures are relatively common injuries that are associated with severe and functionally debilitating pain. ${ }^{1-7}$ The current standard of care for people with sacral insufficiency fractures includes the administration of analgesics of varying efficacy and, in some patients, prolonged bed rest. ${ }^{2-5,8,9}$ It has been reported that sacral insufficiency fractures may require up to 12 months to heal. ${ }^{4,9}$ Prolonged immobilization of elderly patients is known to be associated with significant risks and complications, including pneumonia, urinary tract infections, and pressure ulcers, as well as deep venous thrombosis and associated pulmonary embolus. ${ }^{8}$ Outcomes of conservative management have been mixed in several reported case series, with some studies reporting recovery in all patients, and others reporting a subset of patients with longer-term disability. ${ }^{1,2,6,7,10-15}$ Indeed, one case series describes poor long-term prognoses in most patients who were followed clinically after diagnosis and conservative management of sacral insufficiency fracture. ${ }^{6}$ In that case series, the authors report significant complications of immobility and prolonged hospitalization. ${ }^{6}$ Other case series also report costly prolonged hospitalization, with a mean admission of 21 days in 1 report, and up to 60 days in another report. ${ }^{2,14}$ Functional outcomes after conservative management seem to vary

Received November 2, 2006; accepted November 22

From the Division of Radiologic Sciences, Department of Radiology (C.T.W., C.W.T.M., M.D.B., P.P.M.), Wake Forest University School of Medicine, Winston-Salem, NC; and the Division of Planning, Assessment, and Institutional Research (B.J.M.-W.), Winston-Salem State University, Winston-Salem, NC.

Paper previously presented at: Annual Meeting of the Radiological Society of North America, November 28, 2005; Chicago, III, and at: Annual Meeting of the American Society of Neuroradiology, May 2, 2006, San Diego, Calif.

Address correspondence to P. Pearse Morris, MB, BCh, Division of Radiologic Sciences, Department of Radiology, Wake Forest University School of Medicine, 2nd Floor, Meads Hall, Medical Center Boulevard, Winston-Salem, NC 27157; e-mail: pmorris@wfubmc.edu DOI 10.3174/ajnr.A0561 among case reports. Although some studies report improvements in symptoms, including mobility status, within 3-5 weeks, others report recovery times of several months, with one case series reporting a return to independent mobility after an average of 11 months. ${ }^{11,13,15}$

Sacroplasty, a variant of vertebroplasty, is a relatively new minimally invasive percutaneous procedure that has been described as an alternative to conventional therapy. ${ }^{9,16,17}$ Many patients with sacral insufficiency fractures report decreased pain and increased mobility within hours after sacroplasty ${ }^{9,16,17}$; however, no formal investigation of the longerterm effects of sacroplasty has been conducted. In contrast, several prospective and retrospective investigations of vertebroplasty have reported relatively long-lasting decreases in pain and improvements in the ability to ambulate and perform various activities of daily living (ADLs) after the procedure. $^{18-29}$

Although sacroplasty is a procedural extension of vertebroplasty, it is not as routinely performed, despite the relatively common occurrence of sacral insufficiency fractures. ${ }^{16,17}$ This may be, in part, because of the lack of data regarding functional outcomes after sacroplasty for persons with sacral insufficiency fractures. Therefore, the first goal of the present study was to investigate the self-reported effects of sacroplasty by using a retrospective questionnaire to query for changes in subjective pain, mobility, and ability to perform ADLs after the procedure. A second goal of the present investigation was to compare the clinical outcomes of sacroplasty with the better-characterized effects of vertebroplasty.

\section{Materials and Methods}

Subjects. Fifteen patients were referred to the Interventional Neuroradiology Clinic in the Department of Radiology at Wake Forest University School of Medicine in Winston-Salem, NC, for sacroplasty 
as a result of failure of conservative therapy. All of these patients were found to be appropriate candidates for percutaneous treatment and subsequently underwent a sacroplasty. Each of these patients was eligible to participate in this study. Patients who have had a vertebroplasty were eligible to participate in the present study if their procedure was performed on the same day that a sacroplasty was conducted $(n=32)$, to control for the interval of time posttreatment and operator variables. The Institutional Review Board for research on human subjects at Wake Forest University School of Medicine approved this investigation, and all subjects provided informed consent to participate in the study.

Questionnaire. Subjects were initially contacted by telephone for permission to send a 12-item questionnaire to their home address through the mail. This questionnaire was based on a similar survey, specifically developed to retrospectively investigate the clinical outcomes of vertebroplasty. ${ }^{26}$ The 12 -item questionnaire included questions regarding the date of insufficiency fracture, as well as intensity of pain, mobility or ambulation, and ADLs.

Subjects were required to recall their overall level of pain and ability to ambulate and perform ADLs before and after percutaneous sacroplasty or vertebroplasty. On the questionnaire, pain was measured with an analog scale: 1 , none; and 10 , worst. ${ }^{26}$ Mobility or ambulation was measured with a 5 -point scale: 1 , normal, no pain; 2 , normal, with pain; 3 , limited, with pain; 4 , wheelchair; and 5, bedridden. ${ }^{26}$ The ability to perform ADLs was evaluated with a 5-point scale: 1, able to perform ADLs without pain; 2, able to perform ADLs with mild pain; 3 , able to perform ADLs with moderate pain; 4, able to perform ADLs with severe pain; and 5, unable to perform ADLs because of pain. ${ }^{26}$ Therefore, each item on the questionnaire resulted in an associated score, with a higher number suggesting worse and a lower number suggesting better status before or after the procedure.

Statistics. We assessed the differences between the sacroplasty and vertebroplasty groups on demographic variables of age and time since the procedure with a 2 -tailed Student $t$ test. A $\chi^{2}$ analysis was conducted to assess for sex differences between the sacroplasty and vertebroplasty groups. Finally, we conducted a 2 (time: preprocedure vs postprocedure) $\times 2$ (intervention: sacroplasty vs vertebroplasty) analysis of variance by using each item from the questionnaire as a dependent measure.

\section{Results}

Subjects. A total of 12 (80\%) of 15 patients who had undergone a sacroplasty participated in the study, compared with 21 (65.6\%) of 32 patients who had undergone a vertebroplasty. Two patients who had the sacroplasty were deceased, and 1 patient refused to participate. Five patients who had a vertebroplasty were deceased, 3 refused to participate, and 3 were lost to follow-up.

A total of 11 (91.7\%) of 12 patients with sacroplasty and 17 $(81.0 \%)$ of 21 patients with vertebroplasty completed the entire questionnaire. One patient with sacroplasty and 2 patients with vertebroplasty failed to respond to one item from the questionnaire regarding the ability to perform housework or handiwork before and after sacroplasty or vertebroplasty. One patient failed to respond to 1 item regarding the ability to bathe after vertebroplasty. Finally, 1 patient failed to respond to one item regarding the ability to transfer from a chair after vertebroplasty.

Demographic characteristics are presented in Table 1. There was no statistically significant difference between the

\begin{tabular}{lcc}
\hline $\begin{array}{l}\text { Table 1: Demographic characteristics of sacroplasty and } \\
\text { vertebroplasty groups }\end{array}$ & \\
\hline Characteristics & Sacroplasty & Vertebroplasty \\
\hline Age $^{*}$ & $72 \pm 13$ & $74 \pm 13$ \\
Gender $(n)$ & 1 & 4 \\
Male & 11 & 17 \\
Female & $654 \pm 345$ & $638 \pm 339$ \\
\hline Days since procedure
\end{tabular}

* Data expressed as mean \pm SD.

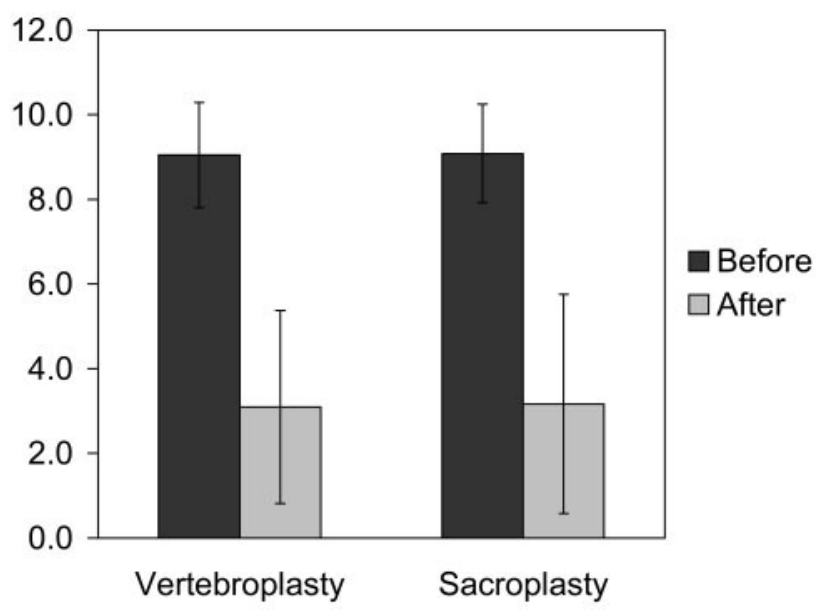

Fig 1. Self-reported fracture-associated pain before and after sacroplasty or vertebroplasty. Patients who had a sacroplasty reported a $65.1 \%$ decrease in fracture-related pain after the procedure. Patients who had a vertebroplasty reported a $65.8 \%$ decrease in fracture-related pain after the procedure.

sacroplasty and vertebroplasty groups in patient age $[t(31)=$ $-0.31, P=.76]$. There was a clear predominance of female patients in both groups, with no significant difference in sex distribution between the sacroplasty and vertebroplasty groups $\left[\chi^{2}(1, n=33)=0.68, p=.41\right]$. No statistically significant difference was identified between groups in the time interval since the procedure $[t(31)=0.13, p=.90]$, which ranged from 254-1268 days (median, 647 days) for the sacroplasty group, and 247-1261 days (median, 634 days) for the vertebroplasty group.

Pain, Mobility, and ADLs. For self-reported scores of overall pain, there was no interaction of procedure $\times$ time $([F$ $(1,62)=0.001, p=.97])$ or main effect of procedure $([F$ $(1,62)=0.01, p=.91])$. There was, however, a main effect of time, such that self-reported scores of overall pain were significantly lower after sacroplasty and vertebroplasty $[F(1,62)=$ 149.32, $p<.001$ ] (Fig 1). Changes in self-reported ability to ambulate and perform each measured ADL also followed this pattern of statistical significance for both the sacroplasty and vertebroplasty cohorts. For the self-reported ADL scores, there was no interaction of procedure $X$ time (walking: $[F$ $(1,62)=1.54, p=.22]$; housework: $[F(1,58)=0.012, p=$ $.91]$; bathing: $[F(1,61)=0.585, p=.45]$; transferring from chair: $[F(1,61)=0.094, p=.76]$; transferring from bed: $[F$ $(1,62)=0.037, p=.85])$. There was also no main effect of procedure on ADL scores (walking: $[F(1,62)=0.353, p=$ .56]; housework: $[F(1,58)=0.541, p=.47]$; bathing: $[F$ $(1,61)=0.002, p=.97]$; transferring from chair: $[F(1,61)=$ $0.886, p=.35]$; transferring from bed: $[F(1,62)=1.820, p=$ $.18])$. However, a main effect of time was demonstrated, such 
Table 2: Ability to ambulate and perform activities of daily living (ADLs) before and after sacroplasty or vertebroplasty*

\begin{tabular}{lccccc}
\hline & \multicolumn{2}{c}{ Sacroplasty } & & \multicolumn{2}{c}{ Vertebroplasty } \\
\cline { 2 - 3 } ADL & Before & After & & Before & After \\
\hline Walking/moving & $3.0 \pm 0.4$ & $2.2 \pm 1.2$ & & $3.1 \pm 0.7$ & $1.8 \pm 1.0$ \\
Housework/handiwork & $4.2 \pm 0.8$ & $2.4 \pm 1.4$ & & $3.9 \pm 1.4$ & $2.2 \pm 1.1$ \\
Dressing & $3.8 \pm 0.6$ & $1.8 \pm 0.8$ & & $3.7 \pm 1.1$ & $1.6 \pm 0.7$ \\
Bathing & $3.5 \pm 0.9$ & $1.8 \pm 0.9$ & & $3.7 \pm 1.3$ & $1.6 \pm 0.7$ \\
Transferring (chair) & $3.8 \pm 0.6$ & $1.8 \pm 1.0$ & & $3.7 \pm 0.9$ & $1.6 \pm 0.7$ \\
Transferring (bed) & $4.0 \pm 0.6$ & $1.9 \pm 1.0$ & & $3.7 \pm 1.0$ & $1.7 \pm 0.7$ \\
\hline
\end{tabular}

* Data expressed as mean \pm SD

that self-reported ADL scores were significantly lower (improved) after sacroplasty and vertebroplasty (walking: $[F$ $(1,62)=25.21, p<.001]$; housework: $[F(1,58)=29.52, p<$ $.001]$; bathing: $[F(1,61)=61.35, p<.001]$; transferring from chair: $[F(1,61)=96.72, p<.001]$; and transferring from bed: $[F(1,62)=89.20, p<.001])$ (Table 2). The lack of a statistically significant interaction of procedure $\times$ time indicates that the improvement in overall pain was equivalent, regardless of which procedure the patient received.

\section{Discussion}

Insufficiency fractures represent the most severe and potentially devastating complication of osteoporosis, a condition affecting millions of people. ${ }^{30-33}$ The incidence of such fractures will likely increase within the rapidly growing population of elderly people as osteoporosis becomes more prevalent. ${ }^{31,32}$ Individuals with insufficiency fractures suffer debilitating pain that often results in loss of functional capacity and diminished quality of life. ${ }^{34}$ The current standard of care for treatment of sacral insufficiency fractures involves pharmacotherapy for pain, and often prolonged bed rest. ${ }^{35}$ In particular, bed rest is known to be associated with such risks as progressive loss of bone mineralization and muscle mass, as well as the development of pneumonia and pulmonary embolism, which may outweigh the benefits of bed rest for some patients. ${ }^{36-40}$ Sacroplasty is a relatively new minimally invasive percutaneous intervention that provides a treatment alternative to current therapeutic options. Several case series have reported near-immediate relief of pain and improvement in mobility after the procedure, similar to reported outcomes of vertebroplasty. ${ }^{9,16,19-22}$

In the present investigation, patients with sacral insufficiency fractures retrospectively reported relatively long-lasting reductions in pain and increases in the ability to ambulate and perform various ADLs after the procedure. Indeed, 1 difference between the present study and the previous investigations of sacroplasty is the reported duration of effects. In one case series, the author reports symptomatic improvement in patients followed for up to 14 weeks after sacroplasty. ${ }^{16} \mathrm{~A} \mathrm{sec}-$ ond case series reports symptomatic improvement in patients followed for up to 9 months post-sacroplasty. ${ }^{9}$ In our investigation, patients reported decreased pain and increased ability to ambulate and perform ADLs for a mean duration of more than 1.5 years (range, 254-1268 days; median, 647 days) after undergoing sacroplasty. To our knowledge, this is the longest follow-up retrospective analysis of the effects of sacroplasty to date.

The present data also suggest that the effects of sacroplasty on pain, mobility, and the ability to perform ADLs are similar to that of vertebroplasty. There was no significant difference between the sacroplasty and vertebroplasty group on the clinical outcomes measured in this study. These findings parallel similar investigations of the retrospective and prospective clinical outcomes of vertebroplasty. ${ }^{18-29}$

Although these data are encouraging, several limitations must be discussed. First, the questionnaire used in the present investigation was based on a similar measure described in a previous retrospective study of vertebroplasty, ${ }^{26}$ which has not been formally validated. This study did not include data from all patients eligible to participate. Missing data from patients who refused to participate, were deceased, or were lost to follow-up could have altered the results of this study.

Intrinsic limitations of a retrospective study, such as recall bias, limited this investigation. It is possible that patient recall was not accurate, and that subjects included in this study overestimated the benefits of their sacroplasty or vertebroplasty because of inaccurate recollection of their initial state. Many well-known factors affect accurate pain recall, including interval of time between patient interviews, and patient age, sex, and current pain status. ${ }^{41-45}$ Some studies have reported poor agreement between prospectively and retrospectively collected data regarding pain and disability. ${ }^{46-48}$ Such studies question the validity of pain recall, especially for long intervals of time between follow-up. However, other evidence suggests that retrospective recall of pain and functional status can be reasonably accurate and, therefore, useful for the evaluation of outcomes, even after extended periods of time. Indeed, one such study that compared more than 100 patients with persistent low back pain demonstrated significant agreement between the initial report of pain and function and retrospective recall, despite a 5- to 10-year interval between collections of data points. ${ }^{41}$ Such data parallels the findings of similar studies, which suggests accurate recall for shorter intervals of time. $^{49}$

In addition to recall bias, it is possible that the patients in the present study simply responded in a manner considered to be pleasing to the investigators, rather than being accurate to their recollection. Indeed, the study design or questionnaire, or both, may have been flawed such that patients favorably rated their outcomes regardless of the treatment rendered (eg, cementoplasty, medications, or other).

This study was limited because of the absence of control groups. Although a conservatively managed group of patients with sacral and vertebral insufficiency fractures exists at our institution, comparison between these patients and our cementoplasty groups would not have been appropriate. At our institution, and at others where sacroplasty is performed, referrals for sacroplasty are often based on failure of conservative management. Many patients with vertebral compression fractures referred for vertebroplasty at our institution also presented after failure of conservative management. Comparison of the present group of patients who had failed conservative management with a group who responded favorably to conservative management would have significantly biased the results. Certainly, a comparison of patients with insufficiency fractures randomized to cementoplasty or conservative management would generate valuable data, especially if conducted in the context of a placebo-controlled and blinded, randomized trial. However, given that clinicians are reluctant to con- 
sider sacroplasty as a first-line treatment for sacral insufficiency fractures over the accepted strategies of conservative management, such randomization is not presently possible at most institutions.

It is important to note that this study was conducted without a sham-procedure control group. As such, the role of the placebo effect as an explanation for the apparent efficacy of vertebroplasty and sacroplasty cannot be excluded. Indeed, only a controlled prospective randomized trial could most accurately establish the true clinical efficacy of sacroplasty and vertebroplasty, beyond the nonspecific effects of placebo. ${ }^{50,51}$ The present study suggests that the effects of sacroplasty are similar to those of vertebroplasty. However, the true clinical efficacy of vertebroplasty is unknown because no controlled, prospective randomized trial of vertebroplasty has been completed to date. ${ }^{25}$

Sacroplasty offers a minimally invasive percutaneous intervention that may rapidly lessen or alleviate pain without sedative and other side effects of pharmacotherapy. Furthermore, sacroplasty may allow faster return to mobility and independence for some patients than pain medication and bed rest alone. Although encouraging, the present results fail to parcel out placebo effects and recall bias, among other limitations. A blinded and controlled prospective randomized trial would be required to address questions regarding the true clinical efficacy of sacroplasty more definitively. Such an endeavor might prove difficult, given the relatively small number of sacroplasties that are conducted each year. Until such a trial can be conducted, studies of the biomechanical effects of sacroplasty with engineering methods such as finite element analysis may provide useful information about the potential mechanical benefits of sacroplasty, because such benefits may contribute to patient reports of decreased pain and increased mobility after sacroplasty.

\section{Conclusion}

Bone cement injections of polymethylmethacrylate have become part of the expertise of neuroradiologists. As such, the development and expansion of the application of this technique will likely be driven by this subspecialty of radiology. The present study suggests that the treatment of sacral insufficiency fractures with sacroplasty is efficacious in relieving pain and improving the ability to ambulate and perform ADLs. These data also suggest that the effects of sacroplasty are comparable with those of vertebroplasty, an accepted and more commonly performed procedure. That sacroplasty seems to substantially decrease pain and morbidity associated with sacral insufficiency fractures may increase interest among neuroradiologists and other interventionalists in providing this procedure more routinely.

\section{References}

1. Lourie H. Spontaneous osteoporotic fracture of the sacrum. An unrecognized syndrome of the elderly. JAMA 1982;248:715-17

2. Grasland A, Pouchot J, Mathieu A, et al. Sacral insufficiency fractures: an easily overlooked cause of back pain in elderly women. Arch Intern Med 1996;156:668-74

3. Lin J, Lachmann E, Nagler W. Sacral insufficiency fractures: a report of two cases and a review of the literature. J Womens Health Gend Based Med 2001;10:699-705

4. Lin JT, Lane JM. Sacral stress fractures. J Womens Health (Larchmt) 2003;12:879-88

5. Taillandier J, Langue F, Alemanni M, et al. Mortality and functional outcomes of pelvic insufficiency fractures in older patients. Joint Bone Spine 2003;70:287-89

6. Dasgupta B, Shah N, Brown H, et al. Sacral insufficiency fractures: an unsuspected cause of low back pain. Br J Rheumatol 1998;37:789-93

7. Tsiridis E, Upadhyay N, Giannoudis PV. Sacral insufficiency fractures: curren concepts of management. Osteoporos Int 2006;17:1716-25

8. Babayev M, Lachmann E, Nagler W. The controversy surrounding sacral insufficiency fractures: to ambulate or not to ambulate? Am J Phys Med Rehabil 2000;79:404-09

9. Garant M. Sacroplasty: a new treatment for sacral insufficiency fracture. J Vasc Interv Radiol 2002;13:1265-67

10. De Smet AA, Neff JR. Pubic and sacral insufficiency fractures: clinical course and radiologic findings. AJR Am J Roentgenol 1985;145:601-06

11. Newhouse KE, el-Khoury GY, Buckwalter JA. Occult sacral fractures in osteopenic patients. J Bone Joint Surg Am 1992;74:1472-77

12. Weber M, Hasler $P$, Gerber $H$. Insufficiency fractures of the sacrum. Twenty cases and review of the literature. Spine 1993;18:2507-12

13. Leroux JL, Denat B, Thomas E, et al. Sacral insufficiency fractures presenting as acute low-back pain. Biomechanical aspects. Spine 1993;18:2502-06

14. Gotis-Graham I, McGuigan L, Diamond T, et al. Sacral insufficiency fractures in the elderly. J Bone Joint Surg Br 1994;76:882-6

15. Aretxabala I, Fraiz E, Perez-Ruiz F, et al. Sacral insufficiency fractures. High association with pubic rami fractures. Clin Rheumatol 2000;19:399-401

16. Pommersheim W, Huang-Hellinger F, Baker M, et al. Sacroplasty: a treatmen for sacral insufficiency fractures. AJNR Am J Neuroradiol 2003;24:1003-07

17. Butler CL, Given CA 2nd, Michel SJ, et al. Percutaneous sacroplasty for the treatment of sacral insufficiency fractures. AJR Am J Roentgenol 2005;184:1956-59

18. Jensen ME, Evans AJ, Mathis JM, et al. Percutaneous polymethylmethacrylate vertebroplasty in the treatment of osteoporotic vertebral body compression fractures: technical aspects. AJNR Am J Neuroradiol 1997;18:1897-904

19. Martin JB, Jean B, Sugiu K, et al. Vertebroplasty: clinical experience and follow-up results. Bone 1999;25(2 Suppl):11S-15S

20. Barr JD, Barr MS, Lemley TJ, et al. Percutaneous vertebroplasty for pain relief and spinal stabilization. Spine 2000;25:923-28

21. Kallmes DF, Schweickert PA, Marx WF, et al. Vertebroplasty in the mid- and upper thoracic spine. AJNR Am J Neuroradiol 2002;23:1117-20

22. McGraw JK, Lippert JA, Minkus KD, et al. Prospective evaluation of pain relief in 100 patients undergoing percutaneous vertebroplasty: results and followup. J Vasc Interv Radiol 2002;13:883-86

23. Perez-Higueras A, Alvarez L, Rossi RE, et al. Percutaneous vertebroplasty: long-term clinical and radiological outcome. Neuroradiology 2002;44:950-54

24. Zoarski GH, Snow P, Olan WJ, et al. Percutaneous vertebroplasty for osteoporotic compression fractures: quantitative prospective evaluation of long-term outcomes. J Vasc Interv Radiol 2002;13:139-48

25. Kallmes DF, Jensen ME. Percutaneous vertebroplasty. Radiology 2003;229:27-36

26. Evans AJ, Jensen ME, Kip KE, et al. Vertebral compression fractures: pain reduction and improvement in functional mobility after percutaneous polymethylmethacrylate vertebroplasty retrospective report of 245 cases. Radiology 2003;226:366-72

27. McKiernan F, Faciszewski T, Jensen R. Quality of life following vertebroplasty. J Bone Joint Surg Am 2004;86:2600-06

28. Trout AT, Gray LA, Kallmes DF. Vertebroplasty in the inpatient population. AJNR Am J Neuroradiol 2005;26:1629-33

29. Ploeg WT, Veldhuizen AG, The B, et al. Percutaneous vertebroplasty as a treatment for osteoporotic vertebral compression fractures: a systematic review. Eur Spine J 2006;15:1749-58

30. Kanis JA, Pitt FA. Epidemiology of osteoporosis. Bone 1992;13 Suppl 1:S7-15

31. Lips P. Epidemiology and predictors of fractures associated with osteoporosis. Am J Med 1997;103:3S-8S; discussion 8S-11S

32. Keen RW. Burden of osteoporosis and fractures. Curr Osteoporos Rep 2003; $1: 66-70$

33. Chang KP, Center JR, Nguyen TV, et al. Incidence of hip and other osteoporotic fractures in elderly men and women: Dubbo Osteoporosis Epidemiology Study. J Bone Miner Res 2004;19:532-36

34. Hasserius R, Karlsson MK, Jonsson B, et al. Long-term morbidity and mortality after a clinically diagnosed vertebral fracture in the elderly-a 12- and 22-year follow-up of 257 patients. Calcif Tissue Int 2005;76:235-42

35. Wild A, Jaeger M, Haak H, et al. Sacral insufficiency fracture, an unsuspected cause of low-back pain in elderly women. Arch Orthop Trauma Surg 2002;122:58-60

36. Bischoff $\mathrm{H}$, Stahelin $\mathrm{HB}$, Vogt $\mathrm{P}$, et al. Immobility as a major cause of bone remodeling in residents of a long-stay geriatric ward. Calcif Tissue Int 1999;64:485-89

37. Baecker N, Tomic A, Mika C, et al. Bone resorption is induced on the second day of bed rest: results of a controlled crossover trial. J Appl Physiol 2003;95:977-82

38. Zerwekh JE, Ruml LA, Gottschalk F, et al. The effects of twelve weeks of bed rest on bone histology, biochemical markers of bone turnover, and calcium homeostasis in eleven normal subjects. J Bone Miner Res 1998;13:1594-601

39. Nishimura Y, Fukuoka H, Kiriyama M, et al. Bone turnover and calcium me- 
tabolism during $\mathbf{2 0}$ days bed rest in young healthy males and females. Acta Physiol Scand Suppl 1994;616:27-35

40. Slipman CW, Lipetz JS, Jackson HB, et al. Deep venous thrombosis and pulmonary embolism as a complication of bed rest for low back pain. Arch Phys Med Rehabil 2000;81:127-29

41. Dawson EG, Kanim LE, Sra P, et al. Low back pain recollection versus concurrent accounts: outcomes analysis. Spine 2002;27:984-93; discussion 994

42. Boyer GS, Templin DW, Goring WP, et al. Discrepancies between patient recall and the medical record. Potential impact on diagnosis and clinical assessment of chronic disease. Arch Intern Med 1995;155:1868-72

43. Carey TS, Garrett J, Jackman A, et al. Reporting of acute low back pain in a telephone interview. Identification of potential biases. Spine 1995;20:787-90

44. Epstein NE, Hood DC. A comparison of surgeon's assessment to patient's self analysis (short form 36) after far lateral lumbar disc surgery. An outcome study [published erratum appears in Spine 1998;23:284]. Spine 1997;22:2422-28
45. Pincus T, Pearce S, McClelland A, et al. Self-referential selective memory in pain patients. Br J Clin Psychol 1993;32:365-74

46. Broderick JE, Stone AA, Calvanese $P$, et al. Recalled pain ratings: a complex and poorly defined task. J Pain 2006;7:142-49

47. Schmitt J, Di Fabio RP. The validity of prospective and retrospective global change criterion measures. Arch Phys Med Rehabil 2005;86:2270-76

48. Pellise F, Vidal X, Hernandez A, et al. Reliability of retrospective clinical data to evaluate the effectiveness of lumbar fusion in chronic low back pain. Spine 2005;30:365-68

49. Bolton JE. Accuracy of recall of usual pain intensity in back pain patients. Pain 1999;83:533-39

50. Turner JA, Deyo RA, Loeser JD, et al. The importance of placebo effects in pain treatment and research. JAMA 1994;271:1609-14

51. Jarvik JG, Deyo RA. Cementing the evidence: time for a randomized trial of vertebroplasty. AJNR Am J Neuroradiol 2000;21:1373-74 